\title{
Evaluating Current Practice and Proposing a System to Enhance Knowledge Assets within a Small Software Development Unit
}

\author{
Sufian Fannoun, John Kerins \\ Department of Computer Science \\ Faculty of Science and Engineering \\ University of Chester \\ Chester, United Kingdom \\ e-mail: s.fannoun@chester.ac.uk, j.kerins@chester.ac.uk
}

\begin{abstract}
Knowledge management and knowledge transfer within organisations challenge continuity and resilience in the face of changing environments. While issues are principally addressed within large organisations, there is scope to evaluate how knowledge assets are managed within small and medium enterprises and to consider how the process might be enhanced. The research reported here aimed to evaluate practice within an evolving software development unit to understand how knowledge has been acquired and utilised to further organisational development. In-depth interviews were carried out with members of the unit to elicit an understanding of individual and collective learning. Qualitative analysis of the data revealed key changes in thinking and practice as well as insight into the development of individuals' contextual knowledge and tacit understanding. This analysis led to the proposal of a bespoke, lightweight web-based system to support knowledge capture and organisational learning. This work is still in progress but it is anticipated that the results will provide a potentially novel and beneficial method for enhancing knowledge assets in small enterprises and consolidating valuable, and potentially scarce, expertise.
\end{abstract}

Keywords- Software engineering; Knowledge engineering; knowledge representation; knowledge management; organisational learning; small and medium organisations; software development.

\section{INTRODUCTION}

The work reported in this paper focuses on the evolution of a software development unit that was established by staff and students within a university computing department in 2009 and which has continued to develop software for a range of clients. The aim of the research project was to evaluate the knowledge acquired by the unit and to identify any key themes emerging from the analysis reflecting organisational learning particularly where such knowledge might be represented and utilised to enhance practice. The potential outcomes of this research will inform a novel and potentially valuable approach to the enhancement of knowledge assets within emerging small enterprises. Work is still in progress and this paper outlines the key steps in the process and illustrates the significant findings so far.

It became apparent that the evolving knowledge, insight and understanding of the practitioners was encapsulated within the range of projects they had undertaken. The nature of the organisation reflected the demands placed upon individuals to engage in a range of demanding tasks in order to meet client requirements within limited time scales. This militated against undue overheads. Consequently the unit tended to minimise documentation. In fact this trend was consistent with the growing tacit and contextual knowledge which members of the unit implicitly shared. These characteristics are likely to feature in other small enterprises in which no formal attempt is made to handle knowledge assets or knowledge transfer.

A proposal has emerged from the analysis conducted so far to capture the knowledge derived from individual projects and to integrate it into a template-based system. This is designed to provide a lightweight support tool to enhance awareness and management of potentially valuable expertise acquired through practice, which can strengthen resilience.

It is anticipated that such an approach can be adopted by other small-scale or start-up enterprises to support organisational learning and development.

\section{MANAGING KNOWLEDGE IN SMALl TO MEDIUM- SIZED ENTERPRISES}

A significant body of literature addresses the nature of knowledge and how it can effectively be captured and exploited within an organisational context. Systematic Knowledge Management (KM) is less likely to be implemented in smaller organisations.

\section{A. Knowledge Management}

The distinction between tacit - generally subjective, context specific know-how - and explicit - generally objective, rational, context free knowledge - is acknowledged [1]. Explicit knowledge can be expressed in writing, formulas and databases for example. Reference [2] discusses the social and economic trends that have led to the emergence of KM and the knowledge-centric view of modern organisations which, if successful, can become an integral element in organisational effectiveness. Issues concerning the value and retention of organisational knowledge and expertise remain significant [3].

Organisations are complex and they represent the generation, utilisation and sharing of tacit and explicit knowledge. This has led to tensions between different perspectives on the value of organisational knowledge [4]. This contrast, between the view of knowledge as a resource that is possessed versus something that is situated in social 
and organisational practices and relationships, is apparent in [5] and [6] respectively.

Reference [7] address the limited effectiveness with which project-based teams are able to utilise knowledge acquired during projects in other projects or other contexts. Overall organisational management also impacts upon KM initiatives, and inevitable political tensions can render aspirations to acquire and share knowledge successfully within organisations difficult to realise [8].

Reference [9] provide comprehensive coverage of types of KM system and their potential organisational impact. A wide range of specialist IT-based systems including Machine-Learning techniques for knowledge discovery [10] as well as technologies such as blogs and wikis can be deployed to support KM initiatives. The ways in which some of these technologies can overcome the perceived limitations of IT systems in capturing and transferring knowledge are addressed by [7] and [11].

\section{B. Small to Medium-sized Enterprises}

According to [12] small to medium-sized enterprises (SMEs) generally differ from larger organisations in that they display the following characteristics: their processes entail simple control systems and planning, and informal reporting; their procedures are less standardised; their structures are less specialised and entail greater multi-tasking and innovation. They also feature a lower degree of bureaucratic management whilst being flexible and focusing more on people within the organisation.

SMEs are considered to play vital role in enhancing the world economy in regard of employment, development, and innovation in addition to generating exports. They may also benefit from adopting KM to harness their experience and help to safeguard their survival[13].However, the implementation of KM initiatives at SMEs is generally low [14]. A number of reasons might account for this: limited resources and budget as well as the ambiguity and uncertainty of adopting and implementing KM [15] or the fact that SMEs' managers are unwilling to devote the requisite effort to long term $\mathrm{KM}$ objectives, for which they find it difficult to establish added value [16].

Reference [17] differentiate between KM tools and KM practices used to support KM initiatives. Evidence suggests that KM practice at SMEs engaged in processes such as capturing, storing, mapping, disseminating, and creating information focus mainly on managing tacit knowledge and people-centred practices: [18], [19], [20], [21] and [22]. SMEs are also likely to adopt informal KM processes, however [23], [24] and [25] stress the importance of formal practices e.g. formal manuals, and knowledge maps.

Specifically with regard to software development organisations, there is evidence that large and small enterprises manage their knowledge assets but this is more prominent in larger organisations. Predictably, [32] indicates that high technology firms have more difficulty managing tacit knowledge than explicit knowledge. According to [33], small software organisations manage their knowledge through teams' continuous communication, which encourages knowledge sharing processes. This is consistent with the behaviour at SMEs in general.

Software development is knowledge intensive and generates intellectual capital as a principal asset [34], therefore, KM can play a significant role. There is evidence of its benefit in facilitating learning, [35], document management, and reusing project knowledge [11]. Not surprisingly, a wide range of software engineering support tools are available, and repositories of shared documents, wikis, collaborative tools, and blogs also facilitate $\mathrm{KM}$ and knowledge transfer in the software development process [36].

\section{RESEARCH METHODS}

The project aimed to gain insight into the knowledge acquired within a small software development unit and to identify key knowledge assets to evaluate how they contributed to the development and success of the unit. A further aim was to determine how the knowledge assets might be utilised in order to enhance practice.

A series of interview questions was compiled and indepth interviews were carried out with each member of the unit. The group comprised six members: the unit manager, two lead developers and the remaining developers, one of whom specialised in design. The questions focused on general themes and contained a series of follow-up questions to probe more deeply into interviewees' experience, observations and viewpoints. The key themes covered: general background and development of the unit; perceptions of the success and limitations of what had been achieved; individual roles and individual progress; details of approaches to software development - e.g. methodologies adopted, issues concerning: risk, quality assurance, change management; and reflections on their individual learning and development within the unit.

The interviews were recorded and transcript files were generated from the audio data. Qualitative research techniques were applied [37], [38], and [39] : key themes were extracted from interviewees' observations and analysed in order to generate an overall representation of the unit's structure and practice. Piecing these details together provided insight into the tacit knowledge that underpinned key knowledge assets, such as dealing with clients for example, and informed the proposal for a system to support knowledge transfer within the unit.

\section{DEVElOPING A MOdEL OF THE UNIT}

Analysis of the interview data revealed a rich source of detail about how the unit had evolved, how individual members had developed their own professional capability, and how factors such as collective experience, adoption of emerging approaches to software engineering, maturing relationships with clients and an improved physical working environment had contributed to the emergence of the unit in its current form.

\section{A. General Evolution}

The unit was established by staff and students within a university computing department. Initially, students 
undertook software development projects for external clients. Membership of the unit fluctuated in the early stages but eventually a core of former students established themselves in key roles and a more coherent unit emerged. The portfolio of contracts has expanded and includes external clients as well as collaborative initiatives with internal partners.

Data analysis revealed key points where practice changed, and identified what was learned at these key evolutionary stages. It also identified the motivation for the change at each of these key points. For example, perceived limitations with the approaches that were being used, or changes in behaviour resulting from previous mistakes and failed projects, or the challenges faced with issues such as code conflicts and program integration. Arguably this has fostered a collective body of tacit knowledge which is now implicit in practice.

Changes in methodological approach, such as the adoption of peer programming in embracing Agile development methods, and improving the physical working environment by occupying an open-plan office, have encouraged collaboration, communication and the sharing of knowledge.

Evaluating the material provided by the interviewees suggests ways in which unit members collectively shaped their own evolving environment. For example, moving away from an initially comprehensive documentation process during development to a much more lightweight approach. This has been accompanied by embracing emerging techniques such as Agile and Scrum. This evolution has seen the unit adapt techniques to meet their own particular requirements and style of working. For example, responses indicated that the demands of Scrum were perceived as onerous for the size of the project teams so methods were scaled down or omitted in order to optimise the utilisation of techniques such as stand-up meetings for example. This has enabled them to establish a successful environment. They have adopted a flexible Agile approach which now incorporates key software support tools. Key knowledge resources reside in contextual practice as well as repositories such as a source code management tool (GitLab), project management software (ActiveCollab), and website analytics software (Sentry).

This general overview of the unit's development and key phases in its evolution led to the high level model of key characteristics at three stages of this process, in which it can be seen from Figure 1 that practice advanced along with changes in the wider software engineering community.

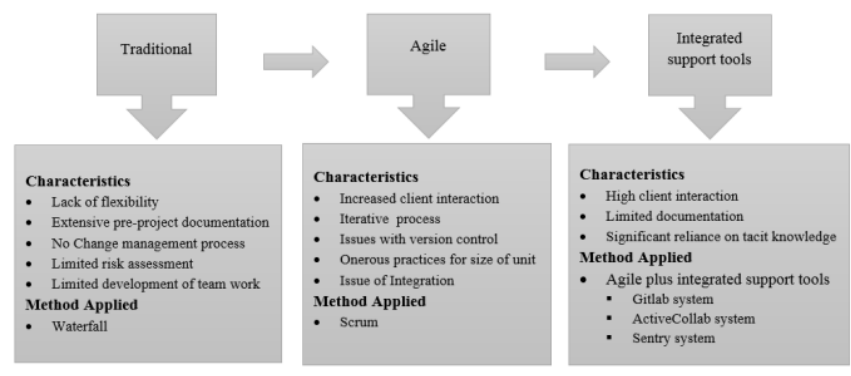

Figure 1. Key phases of the unit's development

\section{B. Individuals' Professional Development}

Individuals have acquired technical skills which have been complemented by the development of soft skills, such as communication, understanding of business processes, resilience, problem solving ability, cooperation and team work, capacity to compromise, self-management, empathy with respect to client relationships, and confidence. These largely tacit attributes contribute to the overall effectiveness of the unit.

The ability to anticipate clients' requirements and behaviour, and then apply this understanding of business processes to interpret the requirements and develop a technical solution is related in the data. Bridging the gap between business processes and a solution represents valuable tacit contextual knowledge, which has been learned and developed through practice.

Analysis of the data pointed up key practices which illustrate how members of the unit have communicated and collaborated to establish tacit business protocols - e.g. an ethos of open communication with clients, which helps mutual understanding of requirements. This mitigates misunderstanding, reworks and client dissatisfaction. Collective decisions to adopt new approaches such as Scrum and the integration of support tools into development processes resulted from informed discussion and engagement with wider software practice. There is evidence of significant progress in learning how to handle clients' projects and to cost and schedule change requests. Members of the unit have to be aware of tensions between software engineering developments, time and cost constraints, and client requirements if the unit is to maintain equilibrium.

\section{A Rationale for Support}

The unit represents a small environment whose members can communicate and work effectively. The analysis revealed that the unit was more fragmented and volatile in the early stages of its development when it encountered difficulties arising from limited experience and a much higher turnover of staff. While a return to such circumstances is unlikely, an attempt to capture valuable expertise and know-how would have potential benefits should staff leave and be replaced by newcomers. Capturing knowledge can also serve as a support for audits and Quality Assurance and to reinforce an understanding of limitations in practice and reasons for adopting new techniques.

The initial outcome of the data analysis is a proposal for a lightweight system to support knowledge capture and dissemination, and organisational learning. The aim is to capture what has been learnt to support current practice concisely without onerous updating requirements.

Unit knowledge is associated with projects and the aim is to encapsulate project knowledge in a generic template. Currently information associated with projects resides in documentation within project support tools e.g. GitLab, ActiveCollab, and Sentry. The aim of the system is to avoid duplication of any data, information, or knowledge from these tools. Template sections will include Management, Technical Details and Project Evaluation. Each of these will provide a concise summary of project characteristics, 
capturing key technical, managerial or other significant factors arising from the project. Links will be provided to relevant documents or components of the support tools where more specific details, e.g. source code comments, can be accessed.

Summary data recorded for some projects will be fairly routine but details of unanticipated difficulties and how they were addressed will be more valuable. By encouraging members of the unit to be critically reflective of current practice, recommendations can be recorded which will articulate viewpoints and will help explain key decisions e.g. recognition of configuration issues mentioned in Section A.

While the proposed repository of project-based templates offers a comprehensive high-level representation of significant project features and associated implications for current approaches to software development, as well as links to relevant project artefacts, there is also scope for more generic tacit knowledge to be made available. There are examples in the data where individuals explain how they have learned to improve their practice. A good example is dealing with clients. This know-how can best be conveyed through a short video clip and it is anticipated that the system will be augmented by integrating relevant clips potentially accessible from a top-level interface with additional links from relevant project pages.

\section{DISCUSSION AND CONCLUSIONS}

Interviews with members of the unit provided a rich source of insight into their knowledge and learning. Analysis of this material has led to the proposal for a system to support the capture of key aspects of this progression. This data collection and analysis marks the first stage of the project. A focus group with members of the unit is now planned in order to verify the observations made so far and to elicit any further comments about their experience and their views on the proposed system. It is anticipated that a prototype system will be built to demonstrate the potential benefits outlined here.

Clearly there are questions about the potential benefits of investing scarce resources in establishing and maintaining a knowledge repository, particularly as the unit has deliberately reduced its reliance on documentation. Issues such as maintaining the currency of knowledge might be raised. The scope of this project has been to gather and analyse data and to propose a feasible utility to support learning and knowledge sharing. Methods to measure the impact of such a utility could be devised to monitor factors such as increased productivity or client satisfaction for example. This would represent a larger study.

An implementation of the current proposal will provide an experimental basis for evaluating the benefits and limitations of the system and offering insights into further enhancements. The principles upon which the proposed knowledge enhancement tool is based are likely to be beneficial in supporting KM initiatives in similar small or start-up enterprises, particularly where valuable skills are in short supply and the continuity of organisational knowledge is critical.

\section{REFERENCES}

[1] Nonaka \& Takeuchi, "The Knowledge-Creating Company Cap 2," Harvard Business Review, no. August, pp. 1-19, 1995.

[2] L. Prusak, "Where did knowledge management come from?," IBM Syst. J., vol. 40, no. 4, pp. 1002-1007, 2001.

[3] D. Leonard, A. Swap, and G.B. Barton, Critical Knowledge Transfer. Boston: MA Harvard Business Review Press, 2015.

[4] D. Hislop, Knowledge management in organisations, $3^{\text {rd }}$ ed. Oxford: Oxford University Press, 2013.

[5] M. Alavi and D. E. Leidner, "Knowledge management systems: issues, challenges, and benefits," Commun. AIS, vol. 1, no. 7, pp. 137, 1999.

[6] A. Lam, "Tacit Knowledge, Organizational Learning and Societal Institutions: An Integrated Framework," Organ. Stud., vol. 21, no. 3, pp. $487-513,2000$

[7] S. Newell, M. Bresnen, L. Edelman, H. Scarbrough, and J. Swan, "Sharing knowledge across projects: Limits to ICT-led project review practices," Manag. Learn., vol. 37, no. 2, pp. 167-185, 2006.

[8] A. McKinlay, "The limits of knowledge management." New Technology, Work and Employment 17(2), 2002.

[9] I. Becerra-Fernandez and R. Sabherwal, Knowledge Management: Systems and Processes, 2nd Edition. Abingdon, Routledge, 2015.

[10] E. Alpaydin, Introduction to Machine Learning, 3rd Edition Cambridge MA: MIT Press, 2014.

[11] I. Rus and M. Lindvall, "Knowledge management in software engineering," IEEE Softw., vol. 19, no. 3, pp. 26-38, 2002.

[12] R. Turner, A. Ledwith, and J. Kelly, "Project management in small to medium-sized enterprises: Matching processes to the nature of the firm," Int. J. Proj. Manag., vol. 28, no. 8, pp. 744-755, 2010.

[13] K. Choochote, "Knowledge Management Strategyfor SMEs," Int. J. Adv. Comput. Sci. Appl., vol. 4, no. 8, pp. 167-172, 2013.

[14] C. W. Chen, M. L. Chang, C. P. Tseng, B. C. Chen, and Y. Y. C. Chang, "Critical human factor evaluation of knowledge sharing intention in Taiwanese enterprises," Hum. Factors Ergon. Manuf., vol. 23, no. 2, pp. 95-106, 2013.

[15] M. R. Lee and Y. C. Lan, "Toward a unified knowledge management model for SMEs," Expert Syst. Appl., vol. 38, no. 1, pp. 729-735, 2011.

[16] M. Baptista Nunes, F. Annansingh, B. Eaglestone, and R. Wakefield, "Knowledge management issues in knowledge-intensive SMEs," $J$. Doc., vol. 62, no. 1, pp. 101-119, 2006.

[17] R. Cerchione, E. Esposito, and M. R. Spadaro, "The spread of knowledge management in SMEs: A scenario in evolution," Sustain., vol. 7, no. 8, pp. 10210-10232, 2015.

[18] C. Wei Chong, S. Choy Chong, and G. Chew Gan, "Inter-organizational knowledge transfer needs among small and medium enterprises," Libr. Rev., vol. 60, no. 1, pp. 37-52, 2011.

[19] I.-C. Lin, R. Seidel, A. Shekar, M. Shahbazpour, and D. Howell, "Knowledge Sharing Differences Between Engineering Functional Teams: An Empirical Investigation," J. Inf. Knowl. Manag., vol. 11, no. 3, p. 1250021, 2012.

[20] S. Massa and S. Testa, "Knowledge domain and innovation behaviour," VINE, vol. 41, no. 4, pp. 483-504, 2011.

[21] J. P. Noblet and E. Simon, "The role of disseminative capacity in knowledge sharing: Which model can be applied to SMEs?," Probl. Perspect. Manag., vol. 10, no. 3, pp. 57-66, 2012.

[22] G. Whyte and S. Classen, "Using storytelling to elicit tacit knowledge from SMEs," J. Knowl. Manag., vol. 16, no. 6, pp. 950-962, 2012.

[23] S. Durst and S. Wilhelm, "Knowledge management in practice: Insights into a medium-sized enterprise's exposure to knowledge loss," Prometh. (United Kingdom), vol. 29, no. 1, pp. 23-38, 2011.

[24] S. Durst and S. Wilhelm, "Knowledge management and succession planning in SMEs," J. Knowl. Manag., vol. 16, no. 4, pp. 637-649, 2012. 
[25] K. Fink and C. Ploder, "Knowledge Management Toolkit for SMEs," Int. J. Knowl. Manag., vol. 5, no. 1, pp. 46-60, 2009.

[26] M. M. Shongwe, "Knowledge management in small software organisations: A South African perspective," in Proceedings of the European Conference on Knowledge Management, ECKM, 2015, pp. 702-708.

[27] S. Basri and R. V. O, "A Study of Knowledge Management Process Practices in Very Small Software Companies," Am. J. Econ. Bus. Adm., vol. 3, no. 4, pp. 636-644, 2011.

[28] D. Panagiotou and G. Mentzas, "Leveraging software reuse with knowledge management in software development," Int. J. Softw. Eng. Knowl. Eng., vol. 21, no. 5, pp. 693-723, 2011.
[29] T. Dingsoyr and D. Smite, "Managing knowledge in global software development projects," IT Prof., vol. 16, no. 1, pp. 26-29, 2014.

[30] A. Menolli, M. A. Cunha, S. Reinehr, and A. Malucelli, "Old' theories, 'new' technologies: Understanding knowledge sharing and learning in Brazilian software development companies," Inf. Softw. Technol., vol. 58, pp. 289-303, 2015.

[31] NJ. Salkind, Exploring Research, 8th Edition . (Ed.). New York, USA: Pearson, 2012.

[32] A. Bryman, Social research methods Bryman. $4^{\text {th }}$ Edition. New York: University Oxford Press Inc, 2012.

[33] D. Silverman, Doing Qualitative Research. A Practical Handbook. $4^{\text {th }}$ Edition. London: SAGE, 2013. 